\title{
Numerical Visualization of Dielectric Barrier Discharge Plasma Actuator Surface Discharge
}

\author{
A. Ahmadi, J. Labadin and P. Phang \\ Department of Computational Science \& Mathematics, \\ Faculty of Computer Science \& Information Technology, \\ Universiti Malaysia Sarawak, \\ 94300 Kota Samarahan, Sarawak, MALAYSIA
}

\begin{abstract}
A single dielectric barrier discharge is a specific configuration for plasma actuators consisting of two electrodes, one coated by a dielectric material and the other is exposed to the air. The purpose of this paper is to study the characteristics of single dielectric barrier discharge (DBD) plasma actuator through numerical modeling. A mathematical model that represents the physical system is presented and the numerical simulations for specific geometry are discussed in this paper. With this model, we can study the physics of the plasma flow. The formulation of the governing equations are divided into two since the physical system can be distinctly separated; one is the electrostatic part and the other is on the fluid flow. The electrostatic part is formulated using the Maxwell's equation which needs to be modified to incorporate the current frequency equation so that the system of equations becomes unsteady which is more realistic compared to a steady system of equations. The fluid flow part is formulated using the Navier-Stokes equations. The equations are re-represented using a vorticity term and stream function so that the flow characteristics can be clearly visualized. All equations are discretized using finite difference method. The discretized equations are then solved using the Gauss-Seidel iteration method. The numerical results show that the vorticity of the plasma is similar in pattern at each time interval. The highest magnitude of the vorticity occurs at the inner part near end of the upper electrode but this magnitude is different at each time. On varying the applied voltage, it is found that the peak vorticity also increases. Therefore, this shows that both the applied voltage and the geometry of the system influence the characteristics of the plasma flow.
\end{abstract}

Keywords: Dielectric Barrier Discharge, Numerical Modeling, Plasma Flow.

PACS: $47.11 . \mathrm{BC}$

\section{INTRODUCTION}

Plasma actuator is a mechanism or a system that produces a plasma layer. One of the plasma actuators is Dielectric Barrier Discharge (DBD). A single DBD is a specific configuration for plasma actuators consisting of two electrodes, one coated by a dielectric material and the other is exposed to the air. Plasma discharge occurs when a high voltage is applied on the exposed electrode. Plasma that produced is distinctive with fluid. The main difference between fluid and plasma is the existing electrostatics force in the plasma. With the existence of this force, the physics of the plasma is different from fluid.

The DBD is commonly studied through experiment (for ex., [1] - [4]) and numerical modeling (see [5] - [7]). Both have their own advantages and disadvantages. The constraint of studying Dielectric Barrier Discharge plasma actuator experimentally is that the physics of the plasma flow could not be studied and obtained directly as it does not produce direct information on it, which is important as far as the efficiency of the plasma actuator is concerned [6]. The motivation for studying the physics of the plasma flow is that the applications of the single DBD have increased in the past few years owing to its ability in controlling fluid flow.

\section{MATHEMATICAL MODELS}

In formulating the system of equations that govern the physics of the plasma flow, two models need to be constructed, i.e. electrostatics model and fluid flow model. Electrostatics model is important in the modeling process because it is one of the characteristics of the plasma that can be used to distinguish plasma from fluid. The system of

Numerical Analysis and Applied Mathematics ICNAAM 2011

AIP Conf. Proc. 1389, 70-73 (2011); doi: 10.1063/1.3636673

(C) 2011 American Institute of Physics 978-0-7354-0956-9/\$30.00 
equations of the electrostatics model was constructed from Maxwell's equations while the system of equations of the fluid flow model was constructed from Navier-Stokes equations.

The formulation of the electrostatics model was done previously as mentioned in [6] where an assumption was made in the modeling process whereby the discharge region is assumed to be in a small scale. This will give the charges of the plasma adequate amount of time to redistribute themselves. Hence, this will reduce the full Maxwell's equations into one set of equation, which is the Gauss law for the electric. The system of equations is

$$
\begin{gathered}
\nabla(\varepsilon \nabla \varphi)=\rho_{c}, \\
E=-\nabla \varphi, \\
f_{b}^{*}=\rho_{c} E=-\frac{\varepsilon_{0}}{\lambda_{d}^{2}} \varphi E,
\end{gathered}
$$

where (1) is an electrical potential equation, (2) is the electrical field equation, and (3) is the electrostatics equation. Here, $\varepsilon$ is the dielectric coefficient, $\varphi$ is the electrical potential, $\rho_{c}$ is the charge density, with $E$ be the electrical field strength, and $f_{b}^{*}$ is the body force which is needed in the fluid flow model. This body force which is also known as the Lorentz body force is in terms of the Debye length $\lambda_{d}, \varepsilon_{0}$ the permittivity with the electrical potential and field strength as in equation (3).

The limitation of the model developed in [6] is that the system of equations is assumed steady. Since an alternating current (AC) varies directly with time, it appears that a steady system is simply unrealistic. To improvise the system, an equation of current frequency is applied to the system as given below

$$
\varphi(t)=\varphi_{\text {peak }} \cdot \sin (\omega t)
$$

where $\varphi(t)$ is the AC voltage function of time, $\varphi_{\text {peak }}$ is the highest applied voltage, $\omega$ is angular frequency and $t$ is time in seconds. Angular frequency, $\omega$, is the scalar measure of rotation rate, which is expressed by,

$$
\omega=2 \pi f,
$$

where $f$ is the physical frequency.

The second model, which is the fluid flow model was formulated by transforming the Navier-Stokes equations into vorticity [8], is given below:

$$
\begin{gathered}
\frac{\partial \vec{w}}{\partial t}+u_{y} \frac{\partial \vec{w}}{\partial x}+v_{x} \frac{\partial \vec{w}}{\partial y}-\frac{1}{R e}\left(\frac{\partial^{2} \vec{w}}{\partial y^{2}}+\frac{\partial^{2} \vec{w}}{\partial x^{2}}\right)+\frac{\partial f^{x}}{\partial y}-\frac{\partial f^{y}}{\partial x} \\
\frac{\partial^{2} \psi}{\partial y^{2}}+\frac{\partial^{2} \psi}{\partial x^{2}}=-\vec{w} \cdot
\end{gathered}
$$

In equation (6), $\vec{w}$ is the vorticity, $R e$ is the Reynolds number of the fluid flow and $f^{x}$ and $f^{y}$ are body forces in $x$ and $y$ direction, respectively. The vorticity of the fluid is related to the stream-function as shown in equation (7) with $\psi$ as the stream-function of the fluid flow.

The system of equations for both the electrostatics and the fluid flow models are unsteady models as there are both depending on time variable which makes these models more realistic.

\section{NUMERICAL FORMULATION}

All equations were discretized using finite difference method. The numerical formulation of the electrostatics model is given in [6]. As for the fluid flow model, the stream-function is dicretized following second-order centered difference method. The convective term in the vorticity equation was discretized using first order backward difference method whereas the diffusive term was discretized using first order centered difference method.

Upon discretization mentioned earlier, the stream-function equation yields to its dicretized form,

$$
\psi_{i, j}=\frac{\Delta x^{2} \Delta y^{2} \vec{w}_{i, j}+\Delta y^{2} \psi_{i+1, j}+\Delta y^{2} \psi_{i-1, j}+\Delta x^{2} \psi_{i, j+1}+\Delta x^{2} \psi_{i, j-1}}{2 \Delta y^{2}+2 \Delta x^{2}} .
$$

While the discretized vorticity equation is

where

$$
\vec{w}_{i, j}^{k+1}=A_{i+1, j}^{k} \vec{w}_{i+1, j}^{k}+B_{i, j}^{k} \vec{w}_{i, j}^{k}+C_{i, j+1}^{k} \vec{w}_{i, j+1}^{k}+D_{i-1, j}^{k} \vec{w}_{i-1, j}^{k}+E_{i, j-1}^{k} \vec{w}_{i, j-1}^{k}+F
$$

